\title{
Surgical Management of Large Occipital Encephalocoele: A Case Report
}

\author{
Sim SKa, Theophilus SC ${ }^{b}$, Noor Azman AR \\ aDepartment of Surgery, Faculty of Medicine and Health Sciences, Universiti Malaysia Sarawak, 94300 Kota \\ Samarahan, Sarawak, Malaysia.

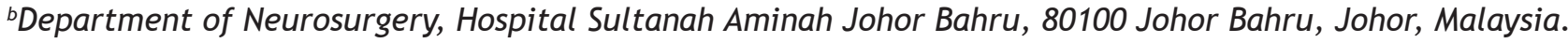

\begin{abstract}
Cranium dysraphism occur less commonly than spinal dysraphism. Overall, occipital encephalocoeles are more frequent than anterior encephalocoes. A large occipital encephalocoele possess difficulty in surgical intervention, not only the sac contains large amount of herniated brain tissue, but the appropriate positioning for successful intubation as well as the higher rate of surgical complications such as cerebrospinal fluid leakage and skin necrosis. In this case report, surgical management of large occipital encephalocoele is discussed. The use of antibiotics in ruptured encephalocoele is recommended.
\end{abstract}

KEYWORDS: Occipital encephalocoele, cranium dysraphism, cerebrospinal fluid leakage.

\section{INTRODUCTION}

Encephalocele is an extension of intracranial structures outside of the normal confines of the skull. The incidence of encephalocele is 1 per 5000 live birth, and it may be associated with partial deletion of chromosome 13q. ${ }^{1}$ Here we report a case of occipital encephalocele associated with syndromic feature and microcephaly, and the surgical intervention.

\section{CASE REPORT}

This baby girl was refered to Neurosurgical team one hour post-delivery. She is the first child from a nonconsanguineous marriage. Antenatal infective screening of mother was negative. At 32 weeks of gestation, transuterine ultrasound demonstrated a swelling at the back of her head. She was delivered by spontaneous vertex delivery as requested by her mother at 37 weeks of pregnancy. The Apgar score was 6 at 5 minutes and 9 at 10 minutes. Her birth weight was $2.7 \mathrm{Kg}$. She has a small and flatten forehead with syndromic features, and the head circumference was $30 \mathrm{~cm}$ with a full but soft anterior fontanelle.

Corresponding author:

Dr. Sim Sze Kiat

Department of Surgery,

Faculty of Medicine and Health Sciences,

Universiti Malaysia Sarawak,

94300 Kota Samarahan, Sarawak, Malaysia.

Tel (h/p): 6019-8945000, Tel(Office): 6082-581000

Fax: 6082-665088

Email: ssksimon@gmail.com
There was a large occipital swelling, measuring $11 \mathrm{x}$ $14 \mathrm{~cm}$, arising from occipital region just below the vertex. It was soft and the trans-illuminating test was negative. There was no skin covering at the dome of the swelling with exposure of underlying meningeal layer and leaking of some haemoserous-like fluid. No high temperature documented since birth. A course of prophylactic antibiotic was initiated in view of ruptured encephalocoele. Otherwise the child was tolerating feeding and moving all four limbs equally with no breathing problem.

Computed tomography (CT) scan of brain showed there was a skull defect at the occipital region (Figure 1). The sac was filled up fully with brain tissue. The intracranial part of brain tissue was not well differentiated and appeared full as well with minimal visible ventricular system.

The child was taken up for surgery after two weeks post delivery. The delay in surgical intervention was due to no ventilator back up in the hospital. The child was intubated in supine position with head supported from below, over the edge of the operating table. After intubation, the child was placed in left lateral position for surgery, with the encephalocoele supported with a head ring (Figure 2). To facilitate painting and draping, encephalocele was gently hold by an assitant with sterile towel.

A "lazy S" horizontal skin incision with circumferential excision of the encephalocele sac was done. The sac was carefully separated from the skin flap. The sac mainly contained mixed necrotic brain tissue and glial tissue. No large vessels were seen during the excision. Water-tight fascioduraplasty was done in view of 
large dural defect. The skin was fashioned and closed primarily (Figure 3, 4).

Post operatively there was no evidence of cerebrospinal fluid leakage or signs of infection. The wound healing was satisfactory. Head circumference charting was continued and clinically there was no signs of raised intracranial pressure. The prognosis of occipital encephalocoele and the possible need of ventriculoparitoneal shunt has been informed to the mother.

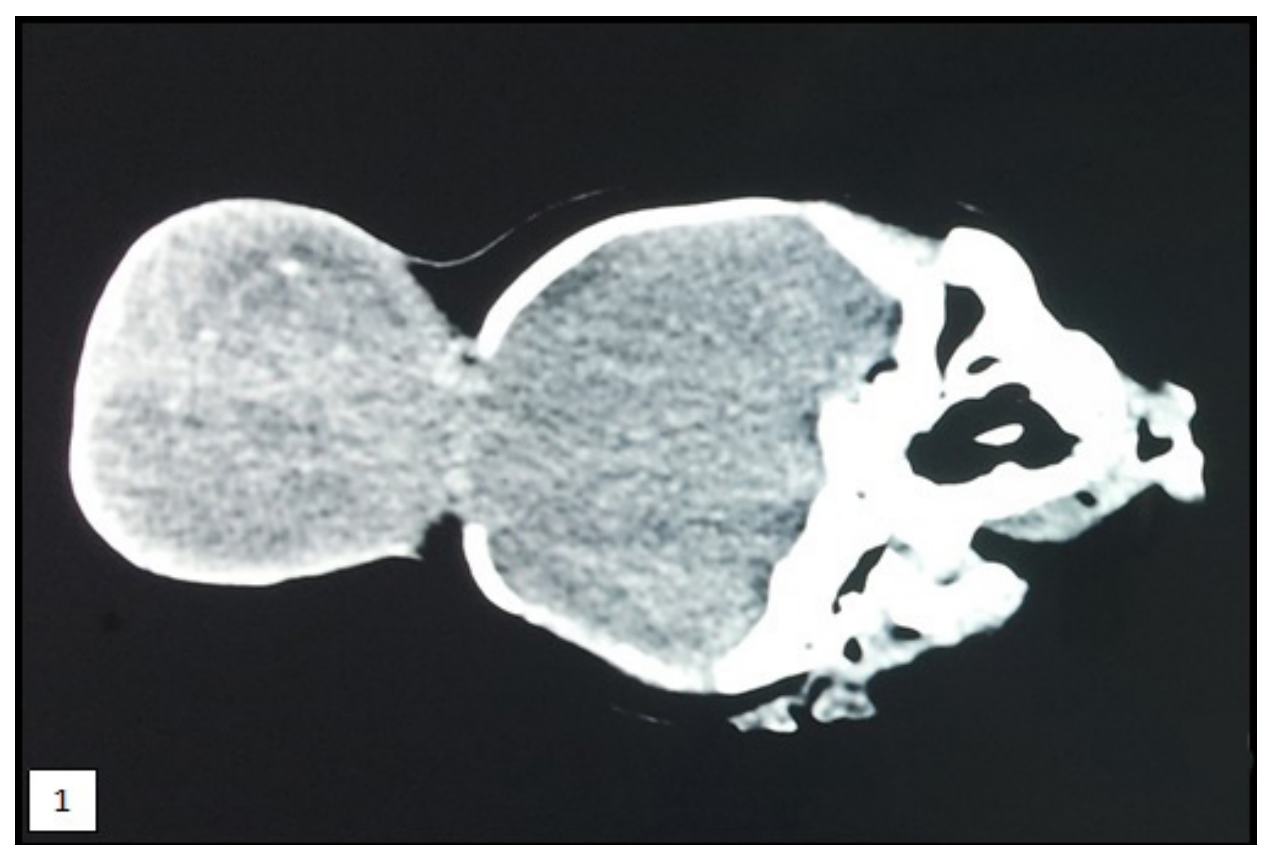

Figure 1. CT scan of brain showed there was a occipital skull defect. The sac was filled up fully with brain tissue. The intracranial brain tissue appeared full as well with minimal visible ventricle system.

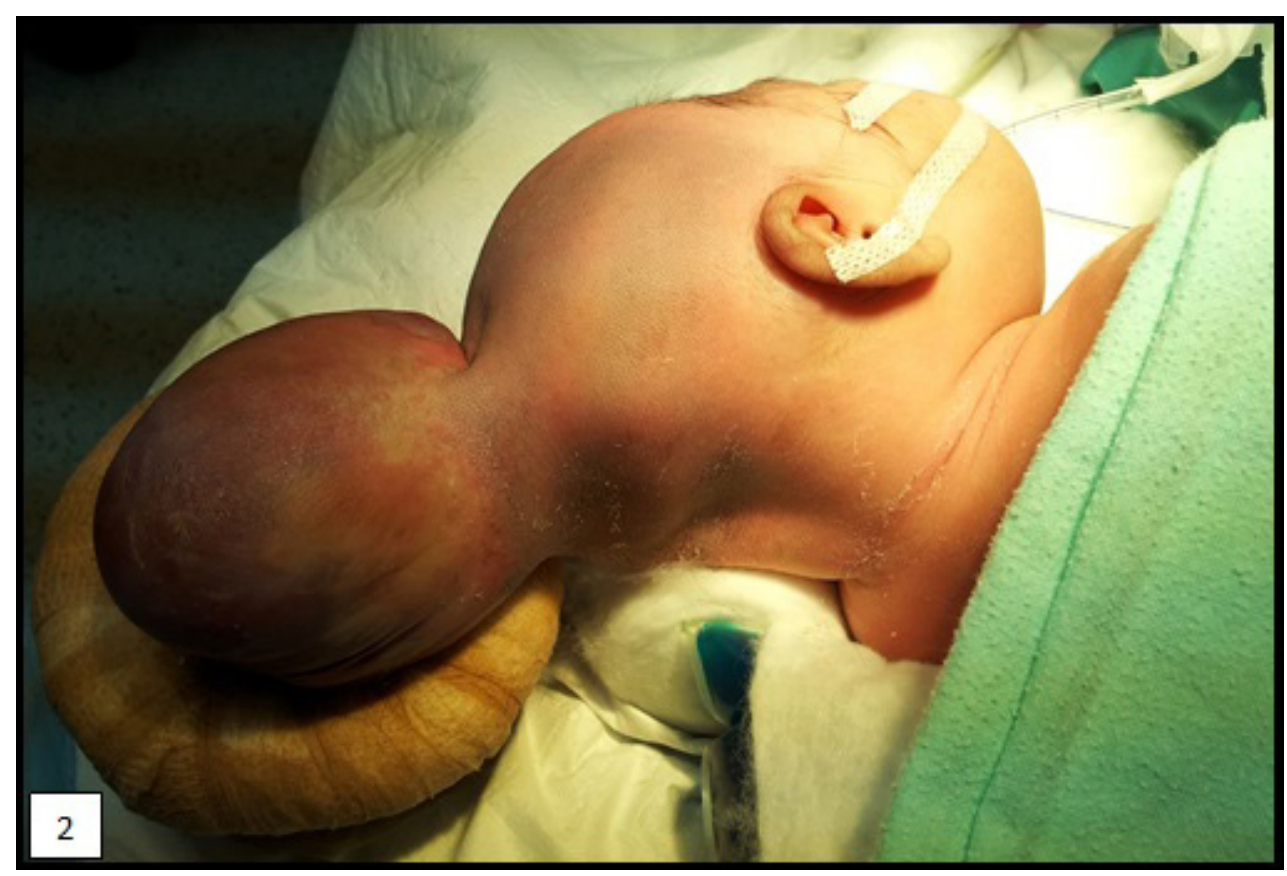

Figure 2. Patient in left lateral position before the surgery. Encephalocoele was supported with a head ring at the edge of the table. 


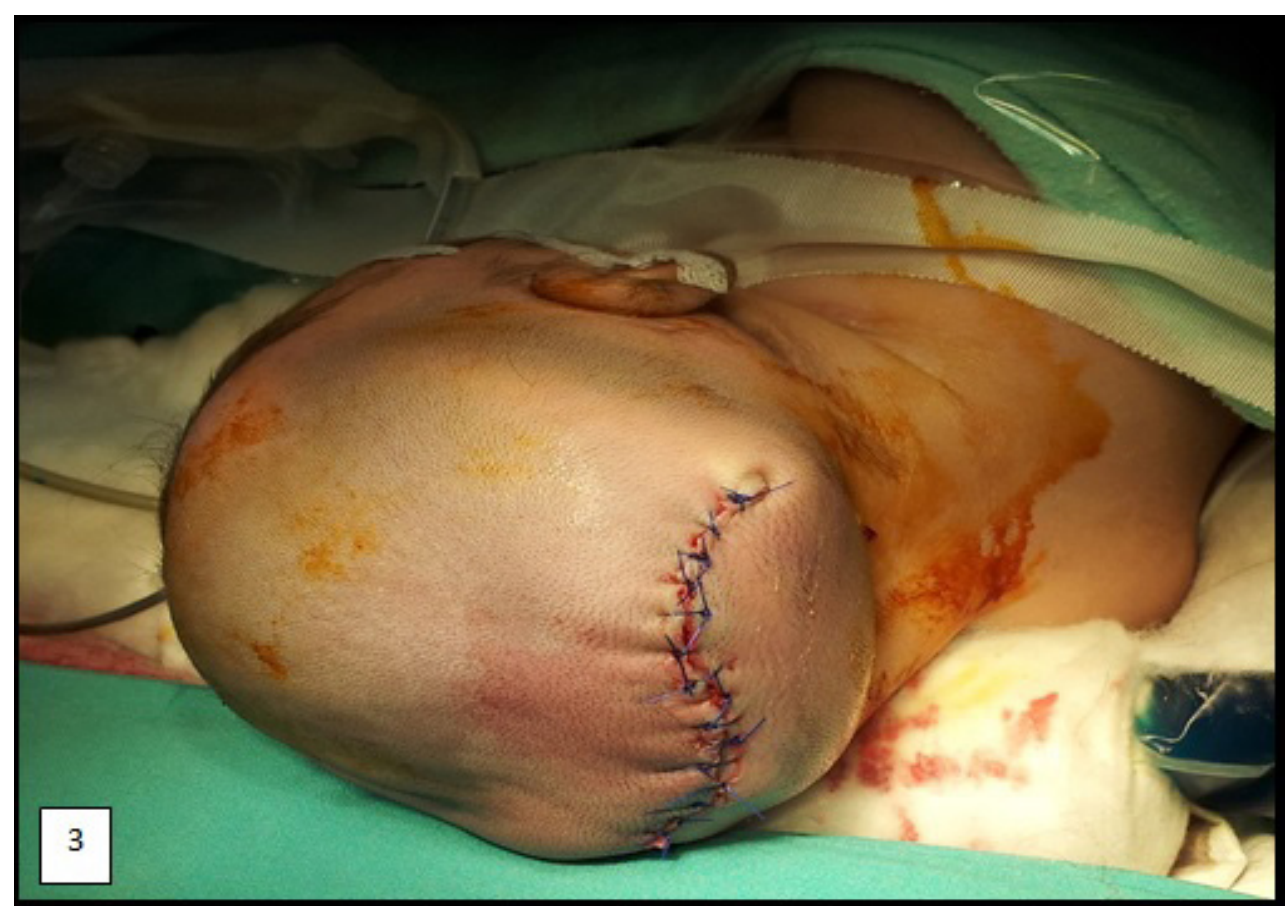

Figure 3. The skin was fashioned and closed primarily. No CSF leakage noted

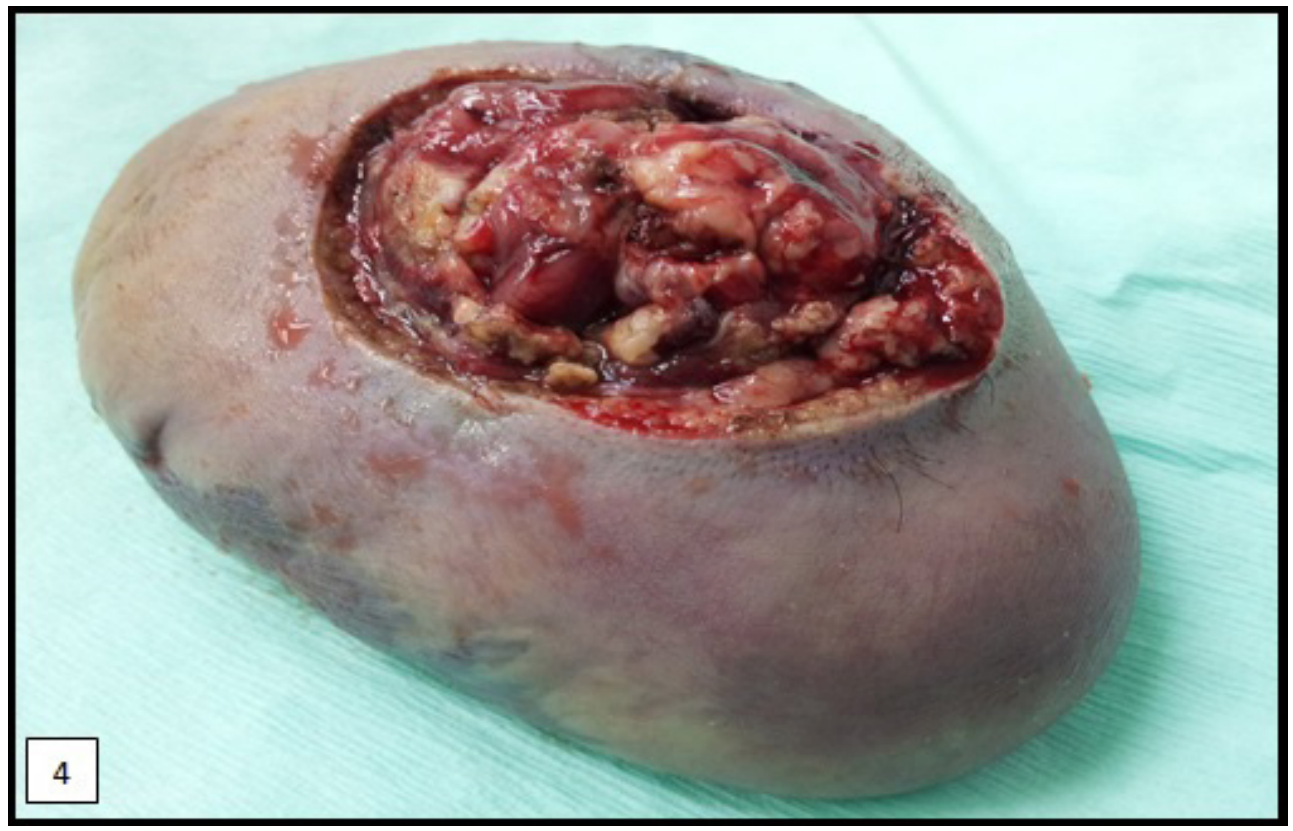

Figure 4. The excised sac with the herniated necrotic brain and glial tissues.

\section{DISCUSSION}

Cranium bifidum is a defect in the fusion of the cranial bone, it occurs in the midline, and is most common in the occipital region. ${ }^{2}$ If meninges and cerebrospinal fluid herniate through the defect, it is called a meningocoele. If meninges and cerebral tissue protrude, it is called encephalocoele. The prognosis is worse if a significant amount of cerebral tissue is present in the sac, if the ventricles extend into the sac, or if there is hydrocephalus. ${ }^{2}$ Folic acid and iron supplementation during pregnancy can prevent this type of congenital anomalies. ${ }^{3}$ Prenatal diagnosis of encephalocele is possible through ultrasonography, maternal serum alfa feto protein (MSAFP) and amniocentesis. ${ }^{4}$
Occipital encephalocele may extend into the foramen magnum and can involve the posterior arch of atlas. When giant encephalocele are associated with microcephaly and micrognathia, it is a predictor of poor outcome. ${ }^{5}$ In these patients, there can be associated lesions including agenesis of corpus callosum, hypoplasia of cerebellum, meningocele, and hydrocephalus. Coexistent hydrocephalus may occur due to torsion of the aqueduct or aqueductal stenosis. Although our patient presented with syndromic features with microcephaly, she has no micrognathia. Her CT scan findings showed undifferentiated part of cerebral tissue with under developed ventricular system. 
Ideally magnetic resonance imaging (MRI) and magnetic resonance venography (MRV) should be done since these are the optimal investigation to visualize the contents of the sac and its relationship to venous sinuses. ${ }^{6}$ The fluid and cerebrospinal fluid (CSF) filled cavities are best demonstrated in MRI scan. Visual evoked response (VER) is of use in giant occipital encephalocele to find out whether the sac contains functional tissue from visual cortex. ${ }^{7}$

Strategy regarding surgical intervention depends upon the amount of neural tissue in the sac, state of CSF pathway, neurological status of the patient and presence or absence of associated congenital anomalies elsewhere in body. ${ }^{5,7}$ Presence of large occipital sac poses difficulty in positioning for intubation in these patients. To prevent any compression and rupturing the sac, the child cannot be positioned supine. Moreover there is limited neck extension as well. Various options for intubation have been reported, suce as intubation in lateral position, or lifting the baby off the table with the help of two assistants. ${ }^{8}$ In our case, the intubation was facilitated by bringing the patient to the edge of the table by a single assistant so that the entire large encephalocele was lying below the table, and the patient was intubated in supine position. After intubation and securing the endotracheal tube, the patient was then put at left lateral position on the operating table with the large encephalocoele supported by a soft head ring.

Anesthetist should be attentive for difficult intubation and careful securing of endotracheal tube in lateral position. Other perioperative problems are serum electrolyte imbalance which may occur during draining of CSF, hypothermia and blood loss. ${ }^{8}$ Sudden loss of CSF during opening of sac may trigger hemodynamic changes such as bradycardia and even cardiac arrest. Thus, continuous arterial monitoring and slow controlled efflux of CSF is recommended.

Intra-operatively, care must be taken to identify the contents of the sac. Rarely, the sagittal sinus, torcula and transverse sinus are in the vicinity of the sac. Major veins should be spared to prevent any venous infarction. ${ }^{7}$ If possible a primary dura closure shoud be made to prevent CSF leakage. In our case, there was a large dural defect where the repair with pericranium was required during the closure of dura. Compromised of the arterial supplies may lead to scalp necrosis. ${ }^{9}$ Hence the incision and closure of the skin should be properly planned before the surgery. For a circular encephalocoele with a small occipital bone defect as in this case, a transverse incision is ideal. In the case where the encephalocoele extends above and below the posterior fossa, then a vertical incision is preferable. Post-operatively the patient should be nursed in prone position to prevent wound breakdown due to compression of the surgical site. Head circumference charting is essential, as the patient may need ventriculo-peritoneal shunt if hydrocephalus developed later due to CSF flow obstruction. Although there was a delay in surgery, our patient was given prophylatic antibiotics and did not show any signs of infection peri-operatively.

\section{CONCLUSION}

Large occipital encephalocoele with syndromic feacture is rare presentation. Prophylactic antibiotic is recommended in ruptured encephalocoele. Specific care must be taken during intubation, cleaning and painting to prevent further rupturing of the sac. Closed monitoring post-operatively is essential as the patient may developed hydrocephalus and required ventriculo-peritoneal shunt.

\section{REFERENCES}

1. Chen CP, Liu FF, Jan SW, Wang KG, Lan CC. Prenatal diagnosis of partial monosomy $13 q$ associated with occipital encephalocele in a foetus. Prenat Diagn 1996; 16:664-6.

2. Nejmi K, Nebi Y, Ismail D, Siddik K. Prognostic factors in patients with occipital encephalocele. Pediatr Neurosurg 2010; 46:6-11.

3. Copp AJ, Greene NDE. Neural tube defects disorders of neurulation and related embryonic process. Wiley Interdiscip Rev Dev Biol 2013; 2:213-27.

4. Chatterjee MS, Bondoc B, Adhate A. Prenatal diagnosis of occipital encephalocele. Am J Obstet Gynecol 1985; 153:645-7.

5. Agrawal A, Lakhkar BB, Lakhkar B, Grover A. Giant occipital encephalocele associated with microcephaly and micrognathia. Paediatr Neurosurg 2008; 44:515-6.

6. Naidich TP, Altman NR, Braffman BH, McLone DG, Zimmerman RA. Cephalocele and related malformations. Am J Neuroradiol 1992; 13:65590.

7. Hoving E, Blaser S, Kelly E, Rutka JT. Anatomical and embryological considerations in the repair of a large vertex cephalocele. J Neurosurg 1999; 90: 537-41.

8. Manhas Y, Chinnan NK, Singh AK. Neonatal airway management in occipital encephalocele. Anesth Analg 2006; 103:1632.

9. Andarabi Y, Nejat F, El-Khashab M. Progressive skin necrosis of a huge occipital encephalocele. Indian J Plast Surg 2008; 41:82-4. 